\section{THE MASTERCLASS SERIES IN FAMILY DOCTOR LEADERSHIP: EVALUATION OF A NEW APPROACH TO LEADERSHIP DEVELOPMENT}

\begin{abstract}
1,2David White* 1,3,4,5Rick Glazier, 1,6Danielle Martin, 1,2Marla Shapiro, 1,6,7 Cynthia Whitehead, 'Sara Crann, ${ }^{1,8}$ June Carroll, ${ }^{1,2,7}$ Risa Freeman, ${ }^{1,9}$ Michael Kidd. ${ }^{1}$ Department of Family and Community Medicine, University of Toronto, Canada; ${ }^{2}$ North York General Hospital; ${ }^{3}$ Canadian Institutes of Health Research; ${ }^{4}$ St. Michael's Hospital, Toronto, Canada; ${ }^{5}$ ICES, Toronto, Canada; ${ }^{6}$ Women's College Hospital, Toronto, Canada; ${ }^{7}$ Wilson Centre, University Health Network, Faculty of Medicine, University of Toronto, Canada; ${ }^{8}$ Sinai Health System, Toronto, Canada; ${ }^{9}$ Flinders University, Adelaide, South Australia, Australia
\end{abstract}

\subsection{6/leader-2019-FMLM.20}

Context Leadership is essential for quality improvement in family medicine.

Objective To assess whether the Master Class approach to developing 'rising stars' in performing arts is effective in developing emerging leaders in academic family medicine.

Design Mixed Methods, combining quantitative evaluation of five sessions and qualitative assessment of participants' precourse assignments and post-course interviews.

Setting The Department of Family \& Community Medicine (DFCM) at the University of Toronto, comprising 14 academic sites, multiple community practices and over 1,700 faculty.Participants: Sixteen 'rising star' DFCM leaders, identified by site Chiefs and Program Directors.

Intervention Five 2-hour evening sessions over ten weeks, each conducted by a different DFCM facilitator with internationally recognized leadership in varied domains.

Outcome measures Qualitative assessment of pre-course descriptions by participants of one of their current challenges, quantitative ratings of each session and qualitative assessment of impact on participants. The problem descriptions and interviews were assessed using descriptive thematic analysis.

Results The participants' descriptions of their leadership challenges revealed significant variation in level of complexity, scope, and framing of the issues. Evaluations of individual sessions were uniformly high, yielding a combined average of all elements of $4.72 / 5$. Analysis of participant interviews at 2-4 months post-course revealed the following themes: impact or potential for impact on their work; most effective aspects; least effective aspects; participant expectations; suggestions for improvement; impact on self-perception as leaders; broader perceptions of leadership approaches; and acquisition of specific skills.

Conclusion The Master Class approach can be adapted to developing rising leaders in family medicine and may be broadly applicable to healthcare leadership development.

\section{TOWARD A MORE INCLUSIVE LEADERSHIP COMPETENCY MODEL AND DEVELOPMENT FRAMEWORK}

${ }^{1}$ Melanie P Standish, ${ }^{2}$ Andrew N Garman. ${ }^{1}$ Illinois Institute of Technology, USA; ${ }^{2}$ Rush University Medical Center, USA

10.1136/leader-2019-FMLM.21

As the pace of health sector transformation continues to accelerate, effective medical and interprofessional leadership continues to increase in importance. Fortunately, there is a growing body of research that can help point the way toward more effective models of leadership and its development. This work delves into a summary of evidence-based guidelines for the 'what' (competencies) and the 'how' (techniques) of healthcare leadership development. The authors aim to help practitioners understand how they can apply a universal framework for organizing leadership development across health professions, understand evidence-based principles for implementing and evolving leadership development programs in healthcare settings, and access free resources that can help them implement effective leadership development initiatives. In summarizing these best practices, the authors intend to create a 'common language' among healthcare professionals in support of interdisciplinary leadership development.

\section{A STEP FORWARD IN CARDIOLOGY ADMISSIONS}

Bupe Chisanga, Andrew Brocklehurst, Akhlaque Uddin. Nottingham University Hospital

\subsection{6/leader-2019-FMLM.22}

Background Majority of the admissions to an acute cardiac unit (ACU) happen out of hours and hence the initial assessments (clerkings) are performed by the out of hours team. Good record keeping is of interest due to patient safety and quality of care delivery. The Royal college of Physicians has set out standards of medical record keeping. This project looked at whether implementing a standardised proforma on the ACU improved the quality of the junior doctor assessment.

Methods A questionnaire was designed and used to assess the general clerking on consecutive admissions. The questionnaire looked at three broad domains that included - patient demographics, patient history and examination, and investigations. Answers to the questionnaire were marked as 'yes', 'no' or ' $\mathrm{n}$ / a'. Following these results, a standardised proforma was designed and implemented. A comparison was then made between general clerking and the standardised proforma. The Results were analysed using the summary independent samples T-test.

Results Nineteen case notes were identified with general clerking; 24 case notes were identified which used the standardised proforma. There was a marked improvement in the clerking with the standardised proforma. There was an increase in documentation of coronary artery bypass graft (CABG) details from $0 \%$ to $100 \%(\mathrm{p}<0.01)$, the smoking history documentation increased from $84 \%$ to $96 \%$ $(\mathrm{p}<0.01)$, the advice given in relation to smoking history increased from $0 \%$ to $40 \% \quad(\mathrm{p}<0.01)$, driving history increased from $11 \%$ to $50 \%(\mathrm{p}<0.01)$, and occupation history increased from $42 \%$ to $75 \%(\mathrm{p}<0.01)$. The junior doctors who used the proforma had overall satisfaction with the proforma with $100 \%$ giving a rating of 3 to 5 out of 5. Also, $75 \%(6 / 8)$ indicated that they would like to continue using it.

Conclusions The implementation of the proforma improved the overall quality of clerking of the admissions to ACU and was received well by the junior doctors. 